\title{
Diseño de un modelo de vivienda bioclimática y sostenible
}

\author{
Ana Cristina Vidal Vidales \\ ana.vidal@utec.edu.sv \\ Luis Ernesto Rico Herrera \\ Guillermo Francisco Vásquez Cromeyer \\ Investigadores Utec
}

\section{RESUMEN}

Se plantea una iniciativa de desarrollo para la población salvadoreña, donde se establece la posibilidad de analizar y desarrollar una vivienda que no solo sea confortable y con beneficios económicos para sus habitantes, sino también amigable con el medio ambiente. El marco teórico contiene criterios y elementos de bioclimatización y sostenibilidad, que posteriormente se adaptan a la realidad nacional, de donde se concluye un potencial para el uso de la energía solar como fuente energética de la vivienda, aprovechamiento de la lluvia como parte del reciclaje de aguas y su climatización, así como la circulación de aire fresco dentro de la estructura propuesta. Se utilizaron tres diferentes experimentos para darle validez al proyecto: la medición de temperatura ambiental, tanto fuera como dentro de tres viviendas ubicadas en diferentes puntos de San Salvador; la elaboración de una maqueta virtual donde se presenta el asoleamiento por horas para visualizar el comportamiento de la vivienda ante la exposición al sol; y un tercer experimento, que trata de la construcción de un túnel de viento y de un modelo a escala para determinar cómo sería la circulación del viento dentro de la vivienda.

Palabras clave: vivienda, desarrollo, sostenible, diseño bioclimático, confort

\section{ABSTRACT}

An initiative of development for the people of El Salvador is established by analyzing and developing a comfortable house that provides economical benefits to the inhabitants, but, at the same time, is environmentally friendly. The project is based on theories that establish criteria and elements of bioclimatic and sustainable architecture, which are later adapted to the national reality, concluding in a potential for the use of solar energy as an energy source for the house, the use of rain as water recycling and for temperature control inside the house, and the circulation of fresh air inside the proposed structure. Three types of experiments where used to support the project: the measuring of internal and external temperatures of three houses in three different locations in San Salvador city; the use of a virtual model to determine the behavior 
of the house to the sun exposure; and a third experiment which consists in the construction of a wind tunnel and a model of the house to determine the movement of the air flow inside the building.

Keywords: house, development, sustainable, bioclimatic design, comfort

Diseño de un modelo de vivienda bioclimática y sostenible

La tradición constructiva de la vivienda salvadoreña se basa en alcanzar procesos constructivos en el mínimo lapso posible y con una estética funcional. Así, pueden observarse urbanizaciones que no toman en cuenta la orientación y distribución adecuada de las viviendas, reduciendo el confort térmico al interior de ellas. La topografía natural de los terrenos es alterada; se construye, con materiales que impermeabilizan el suelo, en áreas naturales protegidas o de servidumbre de ríos y fuentes superficiales de agua, impidiendo que la naturaleza sea capaz de regenerarse por sí misma y dando lugar a desastres naturales, tales como deslaves e inundaciones.

Al diseñar viviendas, las condiciones climáticas se consideran a un nivel práctico y no se profundiza en la eficiencia que el diseño pueda desarrollar para contrarrestar efectos negativos ambientales. Al respecto, Olgyay (2002:10) dice: “[E]l proceso lógico sería trabajar con las fuerzas de la naturaleza y no en contra de ellas, aprovechando sus potencialidades para crear unas condiciones de vida adecuadas".

El aprovechamiento de recursos naturales de cada región, sin implicar su degradación, contribuye a una vida más confortable y armónica, y cuando se combina con una necesidad básica de las sociedades, como lo es la vivienda, el aporte a la vida cotidiana y, por lo tanto, al desarrollo, se vuelve significativo, pues contribuye al aspecto económico -reduciendo costos y demanda de servicios-, y al cultural -evitando la pérdida de respeto por el medio ambiente y creando un espacio más agradable para ser habitado-, entre otros.

\section{Arquitectura sostenible}

Puede decirse que la especialidad de arquitectura sostenible se desprende de la corriente de desarrollo sostenible. En sí, la arquitectura sostenible podría definirse como toda aquella que aprovecha al máximo los recursos disponibles, intentando conscientemente reducir los efectos negativos que sus productos puedan ocasionar al medio ambiente.

Acerca de este último punto, es necesario hacer notar que toda alteración al medio ambiente, incluidas las construcciones, tienen efectos negativos sobre él. Sin embargo, es necesario saber identificar qué tipo de daños se está haciendo para poder resarcirlo en el mismo lugar afectado y su entorno -recordando que se está tratando con el ecosistema, razón por la cual es necesario comprender las relaciones entre todas y cada una de las partes que lo componen-, de manera que el impacto ocasionado, o huella ecológica, se mantenga dentro de un límite que permita al ecosistema su propia recuperación, para que también pueda ser aprovechado por las futuras generaciones. Por lo tanto, uno de los retos de la arquitectura sostenible es lograr un equilibrio entre las edificaciones y el territorio. 


\section{Arquitectura bioclimática}

De acuerdo con la posición de Muñoz (2003), “la construcción bioclimática se preocupa específicamente de la eficiencia energética dentro de la casa, no tanto de los materiales que utiliza y mucho menos de un enfoque ético que involucre las desigualdades sociales". Cabe mencionar que esta definición se apega específicamente a las manifestaciones de arquitectura bioclimática actuales, donde aparentemente el enfoque bioclimático es utilizado para satisfacer las necesidades de ciertos grupos sociales, haciendo uso de tecnologías que muchas veces terminan por representar un daño al medio ambiente y, por lo tanto, dejando de lado el concepto integral de desarrollo.

De acuerdo con Olgyay (2002), el proceso constructivo de una vivienda climáticamente equilibrada se divide en cuatro etapas: 1) Análisis de los elementos climáticos del lugar, 2) Evaluación biológica, 3) Análisis de las soluciones tecnológicas aplicables, y 4) Aplicación arquitectónica.

Adaptación de los criterios de diseño bioclimático y sostenible al contexto nacional: Propuesta de diseño de vivienda.

Conceptualización del proyecto. La vivienda bioclimática y sostenible se puede considerar como una edificación con disposiciones técnicas y estéticas armoniosas con el medio ambiente, que responda físicamente a las necesidades humanas propias del habitar, $y$, al mismo tiempo, brinde el confort térmico para desarrollarlas en el interior y genere menor dependencia de los sistemas energéticos de servicio.

Ubicación del proyecto. Al ser una fase de conceptualización arquitectónica, el terreno donde se emplazaría la vivienda bioclimática y sostenible es ficticio, siendo sus condicionantes dimensionales las necesarias para la proyección arquitectónica. La morfología del terreno se estaría considerando con pendientes de un grado intermedio por ser una característica típica de los suelos de la ciudad de San Salvador.

Usuario. El destinatario de la vivienda propuesta sería una familia nuclear, compuesta por cuatro miembros: el padre, la madre y dos hijos, un índice recurrente en los datos estadísticos que arrojan los censos. El proyecto se enfoca hacia el estrato socioeconómico de nivel medio, pues es el sector que, por lo general, alcanza a satisfacer las necesidades de este bien.

Requerimientos especiales. Por la problemática que generan las condiciones ambientales de la ciudad, el diseño deberá contemplar dos factores: temperatura y dependencia de servicios. De estos se derivan los siguientes requerimientos especiales para direccionar el diseño:

- Bajar el impacto térmico al interior de la vivienda.

- Independizar a la vivienda del régimen eléctrico convencional.

- Disminuir el consumo de agua potable del régimen hídrico convencional.

Plantas de distribución. El prototipo de la vivienda conseguido mediante el proceso de diseño produjo una planta de distribución racionalizada geométricamente de forma rectangular, que posee un área aproximada de $80,93 \mathrm{~m}^{2}$.

La distribución espacial necesitó desarrollar diferentes alturas para separar las actividades, lo que se corrobora 
al observar las leyendas de los niveles en los planos. Se utilizó un medio nivel para dotar al diseño del principio de transparencia, que significa volver el espacio controlable por alcanzar a visualizarse en casi su totalidad.

Los planos realizados se generaron para el sótano y para la primera y la segunda plantas, pero es de hacer notar que, por practicidad, el sótano y la primera planta se manejarán como uno solo y el segundo nivel se observará aparte.

Desde el primer nivel puede observarse que la disposición ortogonal de las columnas acentúa la estructura de la edificación de manera compacta, lo que es deseable, puesto que la zona de El Salvador es altamente sísmica. El área tributaria del marco estructural es de $14 \mathrm{~m}^{2}$.

Figura 1.

Planimetría del proyecto. Primer nivel y sótano

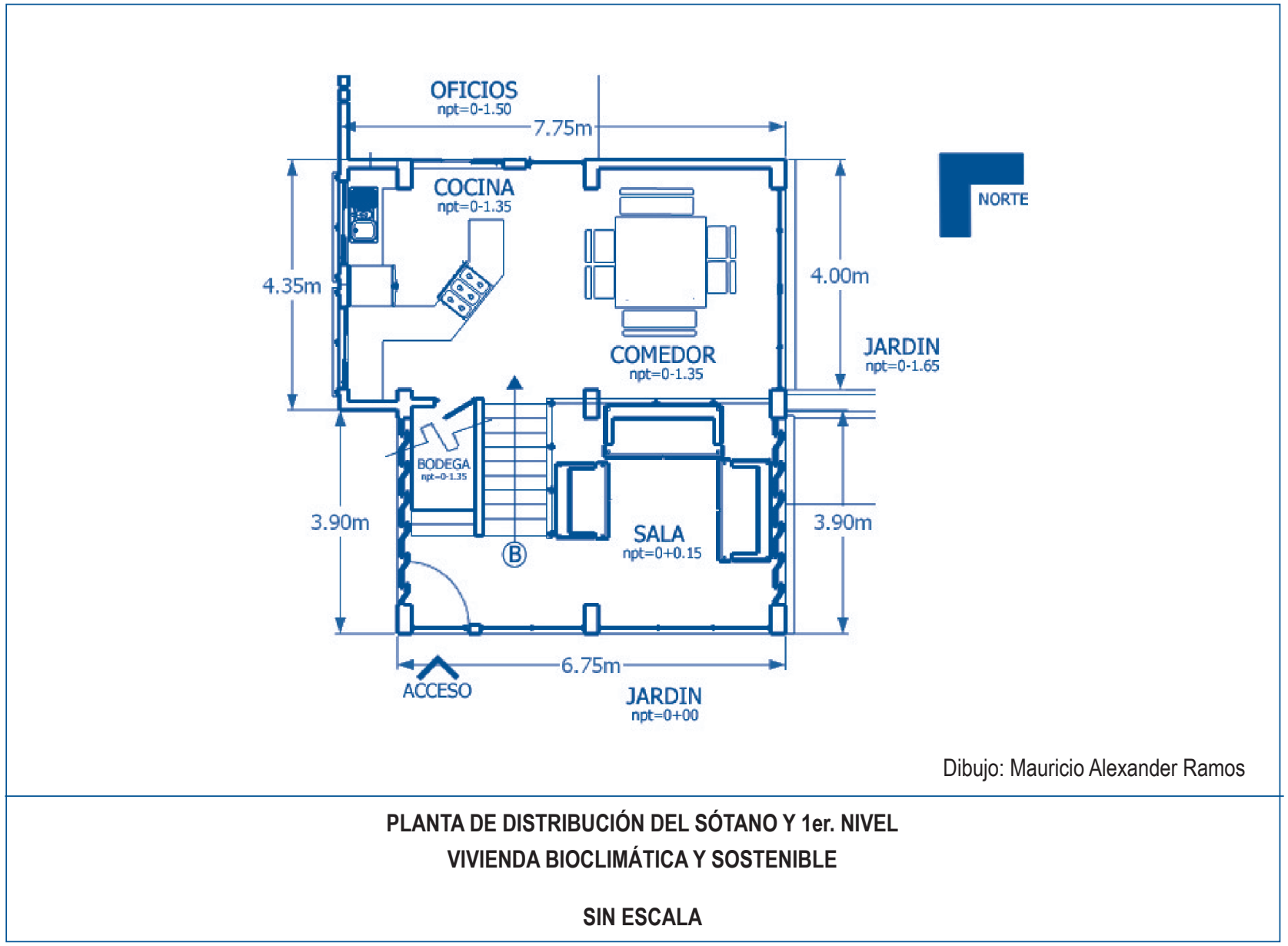

(Nota. Elaboración propia.)

En la planta del segundo nivel, el entrepiso se ha previsto ser diseñado con un sustrato de acero para piso corrugado con acabado de losa de concreto (Allen, 2002), que tiene la ventaja de brindar ligereza a la estructura y su construcción se realiza en un tiempo relativamente corto. 
Figura 2.

Planimetría del proyecto. Segundo nivel

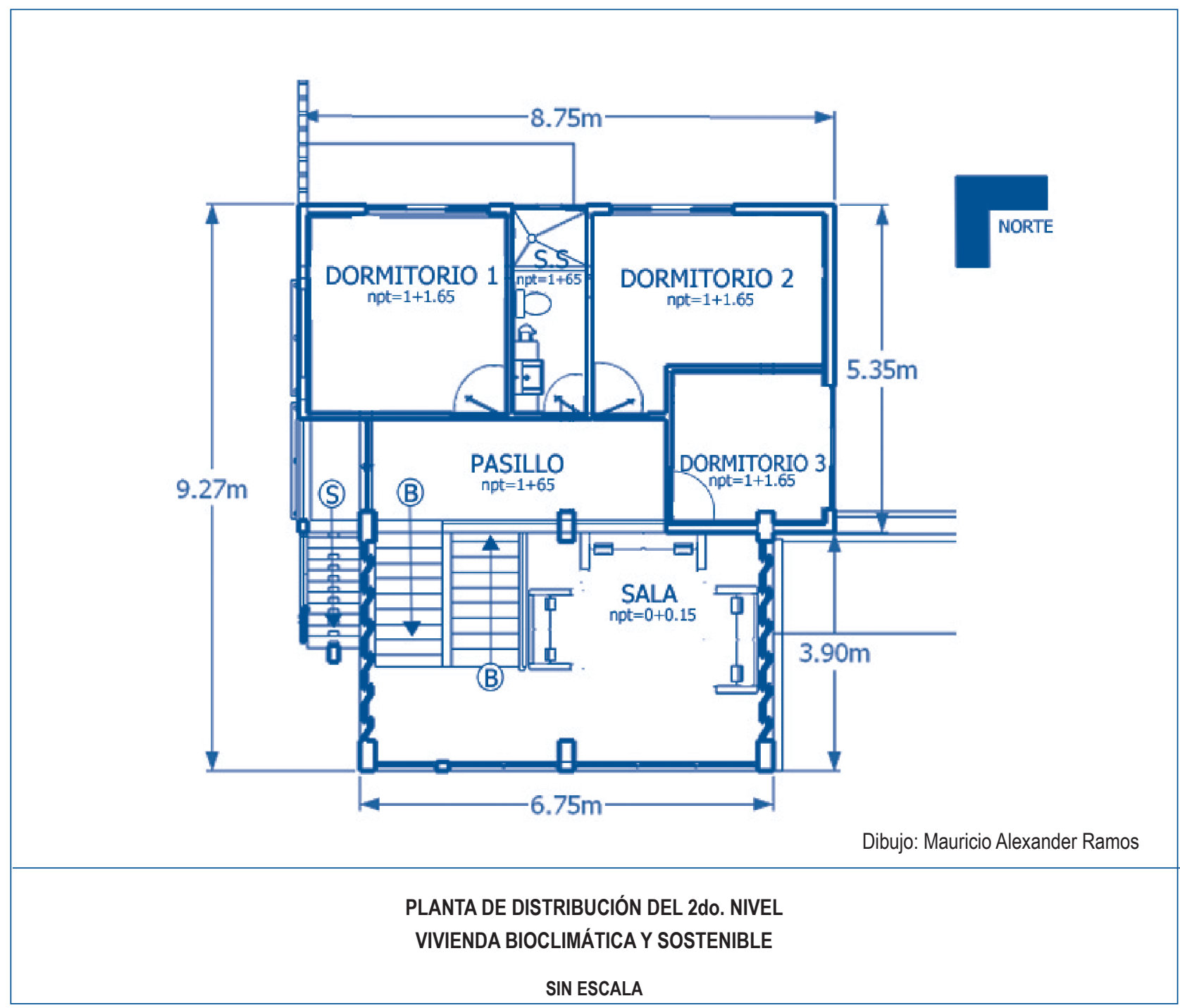

(Nota. Elaboración propia.)

Fachadas. En ellas se pueden verificar las alturas alcanzadas por el diseño, teniendo como altura máxima 6,05 m en el extremo poniente; mientras que en el oriente, por la diferencia de nivel que posee el terreno de emplazamiento, presenta 7,55 m.
Los vanos de las ventanas se han dispuesto siguiendo la regularidad producto del planteamiento ortogonal y cuadrangular practicado en la planta, y su colocación se ha efectuado en sentido nororiente para evitar un fuerte asoleamiento. 
Figura 3.

Perspectiva aérea No. 1

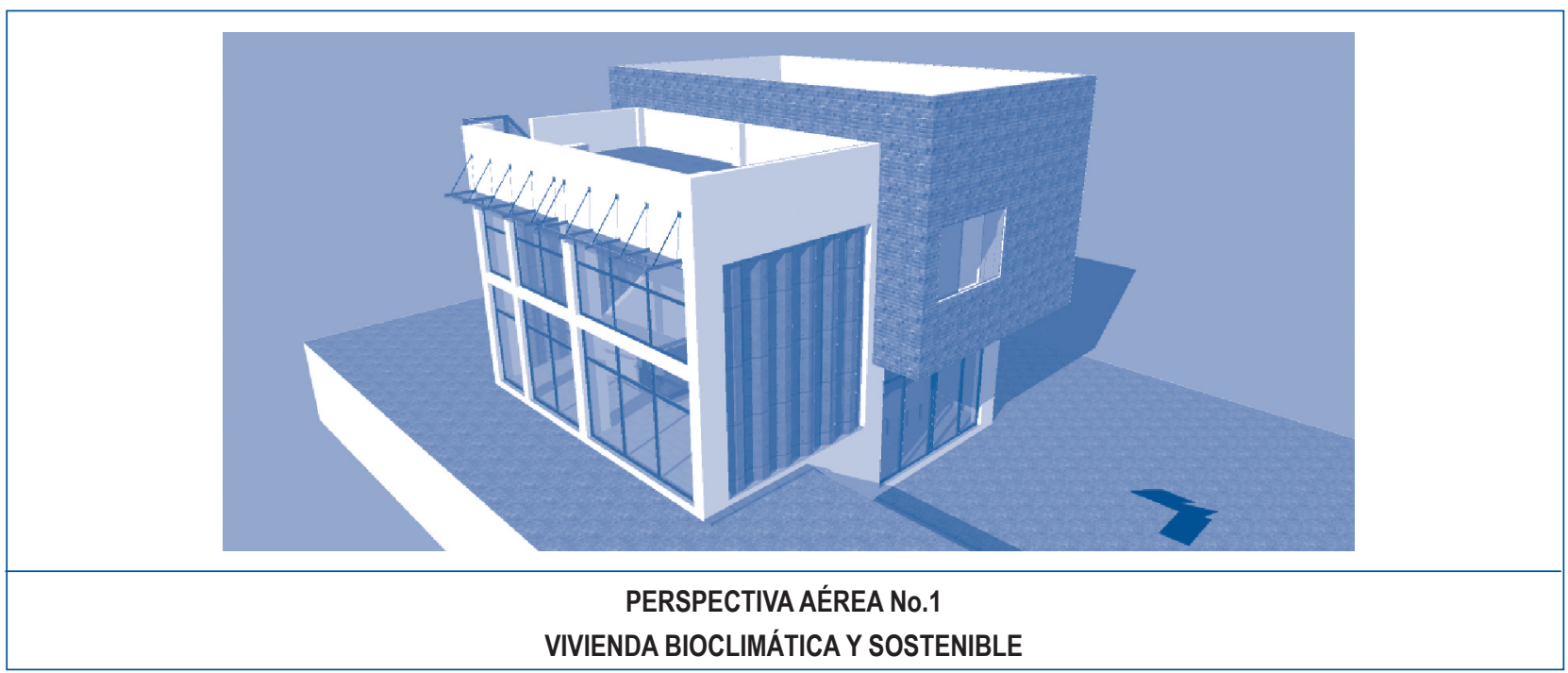

(Nota. Elaboración propia.)

Figura 4.

Perspectiva aérea No. 2

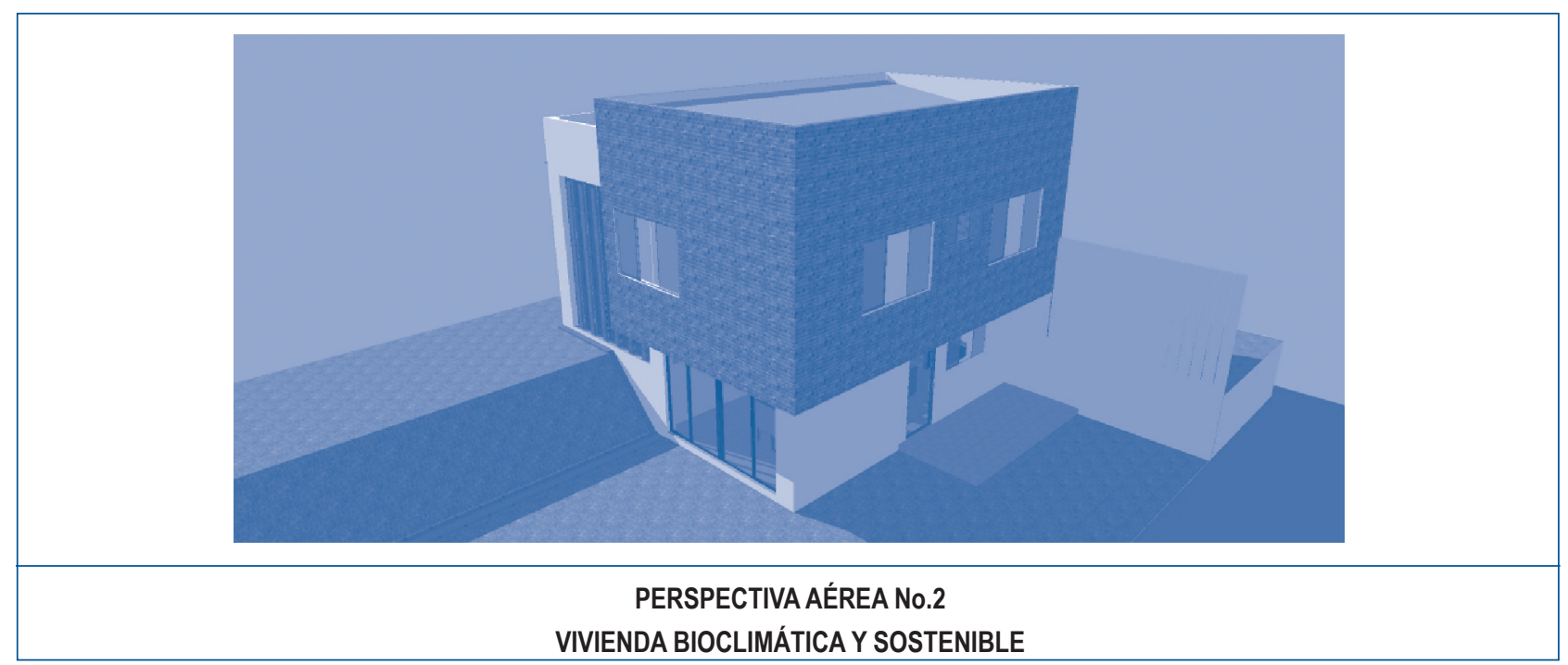

(Nota. Elaboración propia.) 


\section{Método general}

Partiendo del marco teórico, donde se plantean los conceptos y criterios necesarios para el diseño de una vivienda con características de bioclimatización y sostenibilidad, se procedió a proponer el diseño de una vivienda que cumpliera con dichos criterios. Cabe mencionar que el marco teórico incluye además una aproximación teórica de los aspectos tratados previamente al contexto nacional.

Para ampliar la información de los aspectos climáticos del país se recurrió a distintas fuentes bibliográficas, así como a contactos en el Servicio Nacional de Estudios Territoriales (Snet), quienes proporcionaron dos valiosos documentos que se convirtieron en una herramienta importante al momento de decidir el tipo de tecnología que se propondrá en el diseño de la vivienda.

Producto del marco teórico planteado y descrito anteriormente, surge una propuesta de diseño de una vivienda unifamiliar, que integra algunos criterios y elementos de diseño tanto bioclimático como sostenible. La vivienda está planteada para el área urbana, con usuarios de estrato social medio.

Es necesario comprobar si esta propuesta es funcional y cumple con los propósitos u objetivos establecidos en la presente investigación, por lo que se recurrió a hacer una serie de pruebas que se describirán más adelante.

\section{Objeto de Estudio}

El objeto de estudio consistió en un modelo de vivienda que fue concebido con las siguientes características:
Criterios de diseño.

1. Ubicación con respecto al sol: eje ortogonal de la vivienda con una rotación de $45^{\circ}$ hacia el oeste, respecto al norte para reducir las temperaturas extremas.

2. Ubicación con respecto al norte geográfico: diseño de paletas tipo "S", colocadas de manera seriada, a una distancia relativamente corta para crear un canal de enfriamiento al hacer pasar con mayor rapidez el aire por el sifón simulado, aprovechando de esta forma las corrientes de viento que afectan a la vivienda.

3. Nivel de confort térmico: doble altura del techo sobre las áreas de uso común, como la sala y el comedor, donde hay mayor aglomeración de personas en una vivienda, permitiendo mayor movimiento de aire, lo que a su vez contribuye a reducir la temperatura extrema.

4. Topografía quebrada del suelo de la ciudad de San Salvador: edificación diseñada con una parte semienterrada, que sirve para la distribución de los espacios internos y a la vez, para proteger pasivamente del calor.

Sistemas tecnológicos aplicados al diseño.

1. Sistema de enfriamiento pasivo. Celosía en paletas tipo sifón: diseño de paletas tipo "S" y colocadas de manera seriada, a una distancia relativamente corta para crear un canal de enfriamiento.

2. Sistema de enfriamiento pasivo. Losa verde: Iosa de cubierta elaborada con materiales impermeables que permiten colocar tierra y engramado para desarrollar un jardín colgante. 
3. Sistema de reducción del asoleamiento. Aleros metálicos: pantalla metálica adosada a la fachada de la edificación donde existe más vidrio, para evitar que los rayos solares ingresen directamente al recinto.

4. Sistema de alimentación eléctrica. Celdas fotovoltaicas: tecnología para la captación de energía solar que se colocaría en el techo de la vivienda, para evitar y/o reducir el consumo de la red domiciliar habitual.

5. Sistema de captación de aguas Iluvias. Red de captación y cisterna: tuberías que ayudan a almacenar el agua en una cisterna, de la cual se puede reutilizar el agua en tareas que no necesiten calidad potable.

\section{Procedimiento general}

Para esta etapa de la investigación, se realizaron tres diferentes tipos de experimentos con la finalidad de identificar, en primer lugar, las temperaturas y aspectos del confort térmico en distintas zonas del área metropolitana de San Salvador, y en segundo lugar, si el diseño de la vivienda era funcional y cumple con los criterios establecidos anteriormente. Para ello se utilizaron distintas herramientas e instrumentos de naturaleza tanto tecnológica como simulación de ambientes, tanto virtuales como reales. La información o datos se recolectaron por medio de tablas y fotografías, según el tipo de experimento.

\section{Experimento 1}

Este experimento consistió en la recolección de datos de temperatura en distintos sectores del área metropolitana de San Salvador, que incluyeron la colonia Buenos Aires, colonia Escalón y los Planes de Renderos. Los sitios se seleccionaron por conveniencia de los investigadores que formaron parte del equipo, debido a que las mediciones debían ser en el mismo lugar, por una semana, a distintas horas del día.

Por motivos de conveniencia, se realizaron dos tipos de mediciones: durante la semana del 25 al 31 de octubre de 2010 a las $22: 00$ hrs, y el día 31 de octubre de 2010 a partir de las 6:00 hrs hasta las 24:00 hrs a intervalos de 2 a 4 horas, según las variaciones de temperatura fueran más perceptibles.

Con este experimento se persiguió ver la diferencia entre las temperaturas internas y externas de una edificación, así como el porcentaje de humedad presente en el ambiente, con la intención de sentar un precedente para la siguiente etapa de la investigación, donde se planea identificar los materiales ideales para el contexto salvadoreño al tratarse de viviendas bioclimáticas y sostenibles.

\section{Instrumentos}

Se utilizaron tres termómetros digitales - uno en cada sitio seleccionado- que proporcionan datos de temperaturas máximas y mínimas cada 24 horas, tanto del interior como del exterior de las edificaciones donde se localicen. Asimismo proporcionan el dato de la temperatura -interna y externa del edificio- en tiempo real.

Los datos recolectados fueron organizados en tablas, para poder sacar un promedio simple que permitiera visualizar las diferencias y similitudes de temperatura entre cada una de las ubicaciones seleccionadas.

Es necesario aclarar que las mediciones fueron hechas por personas distintas en un horario en el que se supuso que 
cada una de ellas tendría acceso al termómetro. Debido a esto, no se descartan errores o fallas de cualquier índole en la medición, toma de datos y transcripción al formato.

\section{Resultados y discusión}

De los datos obtenidos para este experimento se concluye que las temperaturas, tanto al interior como al exterior de las edificaciones, son distintas debido a las propiedades aislantes de los materiales con las que están construidas. Sin embargo, no puede afirmarse con seguridad cuál de las dos es más alta, si la interior o la exterior, debido a que los datos entre cada una de las mediciones varían. Esto puede deberse a la acumulación y refracción de calor del mismo material a la hora en la que fueron hechas las mediciones.

Otro factor que afecta las mediciones es el microclima de la habitación en la que se colocó el termómetro, ya que este depende de su ubicación en la vivienda, la cantidad de aire que circula dentro de ella, así como la ubicación de la vivienda en sí, entre otros. En general puede concluirse que los porcentajes de humedad más altos se obtuvieron en las colonias Buenos Aires y Escalón, así como las temperaturas promedio más altas.

Cabe mencionar que durante la semana en que se realizaron las mediciones hubo muchas variaciones climatológicas, desde precipitaciones pluviales hasta vientos, pues las mediciones se llevaron a cabo durante el período de la transición de la estación lluviosa a la seca. Esto puede significar que las mediciones ilustren casos muy específicos de ciertas circunstancias climáticas y no necesariamente un patrón que vaya a repetirse con seguridad durante un período de tiempo. Sin embargo, el pequeño ejercicio permitió una visualización general de cómo afectan la temperatura interna de una edificación aspectos tales como ubicación geográfica, materiales de construcción de la edificación y condiciones topográficas del terreno específico, entre otros.

\section{Experimento 2}

La finalidad del experimento 2 fue proveer una visualización de cómo la trayectoria del Sol afectaría al diseño de vivienda propuesto. Este experimento se llevó a cabo en una modalidad virtual debido a que la realización de este experimento en forma física requería de recursos con los que, por la naturaleza de la investigación, era difícil contar.

La simulación virtual permitió recrear las condiciones de asoleamiento y proyecciones de luz y sombra en el diseño propuesto, específicamente en las fechas de los solsticios y los equinoccios del año 2010: equinoccio de 20 de marzo, solsticio de 21 de junio, equinoccio de 23 de septiembre y solsticio de 21 de diciembre. Se seleccionaron estas fechas porque es en ellas donde se da lugar a los cambios más importantes en el ángulo y posición del Sol con respecto al planeta a lo largo del año. Estas fechas definen las estaciones y la cantidad de horas de sol que se reciben durante el día.

Las simulaciones se realizaron a cada hora para las 6:00 hrs hasta las 18:00 hrs.

\section{Instrumentos}

Tomando como base las representaciones gráficas ya elaboradas del diseño propuesto, se utilizó el programa Google SketchUp Pro 8, con sus herramientas de creación de escenas y estudio de sombras, donde se colocaron los datos de ubicación de la región (latitud y longitud), la zona horaria y las horas en las que se deseaba visualizar un resultado. 


\section{Resultados y discusión}

Los resultados se presentaron en una visualización, tanto en perspectiva como en planta, de manera que es posible observar mejor el comportamiento y las proyecciones de las sombras a lo largo del día en el modelo virtual.

Tabla 1.

Ejemplo de la proyección de sombras en el diseño propuesto para el día 20 de marzo de 2010 (equinoccio)

\section{Figura 5.}

Vista en perspectiva del modelo a las 7:00 hrs

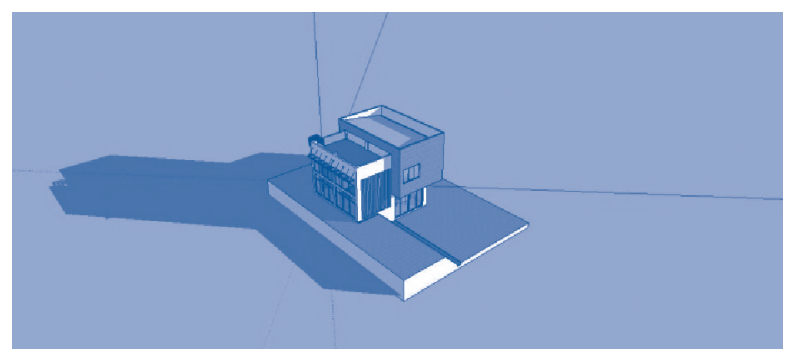

(Nota. Elaboración propia.)
Figura 6.

Vista en planta del modelo a las 7:00 hrs

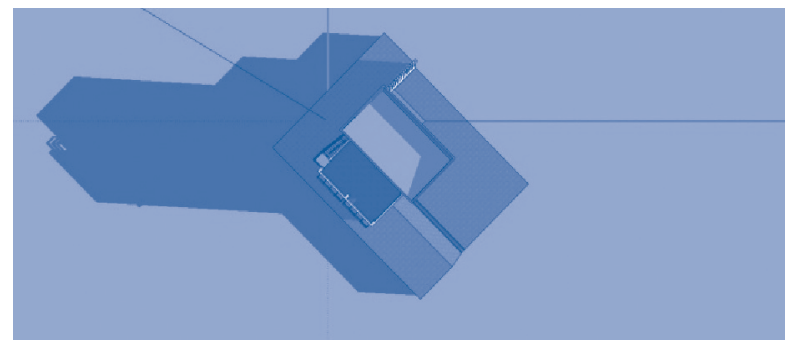

(Nota. Elaboración propia.)

(Nota. Durante el equinoccio del mes de marzo, el sol aún no ha salido a las 6:00 hrs, por lo tanto las sombras comienzan a proyectarse después de esa hora. Elaboración propia.)

Partiendo de estos modelos de simulación virtual, es posible observar que la orientación de la vivienda a $45^{\circ}$ al oeste, con respecto del norte, efectivamente contribuye a reducir los efectos de la radiación solar directa, ya que ninguna de las fachadas está completamente expuesta al sol. Las fachadas que pueden ser más afectadas por la radiación directa poseen menos aberturas y estas son de menor tamaño.

Por otro lado, estos modelos de simulación ayudan a identificar cuáles son las fachadas que necesitarían de un tratamiento de barreras naturales -como por ejemplo la fachada principal- para reducir, en la medida de lo posible, que el sol de la tarde produzca una sensación de calor excesivo al interior de la vivienda. También es posible identificar, por medio de esta técnica, otros elementos de ambientación que permitirán complementar el proceso de bioclimatización de la vivienda.

\section{Experimento 3}

Este experimento consistió en la simulación de la trayectoria del viento a través de los distintos elementos de la vivienda propuesta. Cabe mencionar que en el país no hay ningún laboratorio que realice este tipo de pruebas, razón por la que fue necesario crear un entorno de simulación para la finalidad que se buscaba, por lo que se construyó un modelo a escala, tanto de la vivienda propuesta como de un túnel de viento que permitiera visualizar la circulación del aire dentro de la vivienda. 


\section{Instrumentos}

\section{Maqueta}

Se elaboró un modelo a escala de la vivienda propuesta con el fin de observar cómo el aire se trasladaría dentro de las distintas habitaciones establecidas. La maqueta se realizó a una escala de 1:30, en acrílico de $1 /{ }_{8}^{\prime \prime}$ de grosor y acetato rígido, unido con silicón transparente, ya que su transparencia permitiría observar mejor el flujo del aire al interior del diseño. Otro elemento que se utilizó para mejorar la visualización del flujo del aire fue un fondo negro en los pisos de la maqueta y en los costados del túnel de viento.
La construcción de las celosías de ambas fachadas laterales resultó dificultosa debido a la escala y al material utilizado, por lo que en la maqueta se representan por medio de cortes longitudinales inclinados, logrando con ello simular el efecto que la celosía produce, aunque el flujo del aire se ve magnificado en las pruebas. Esto último permitió una mejor visualización del flujo del aire al interior del modelo, aunque no representa la magnitud real de este por motivos de escala.

La base de la maqueta fue elaborada con capas de poliestireno expandido (durapax) de 3/4" de grosor para de esta manera lograr la topografía determinada en el diseño de la vivienda propuesta.

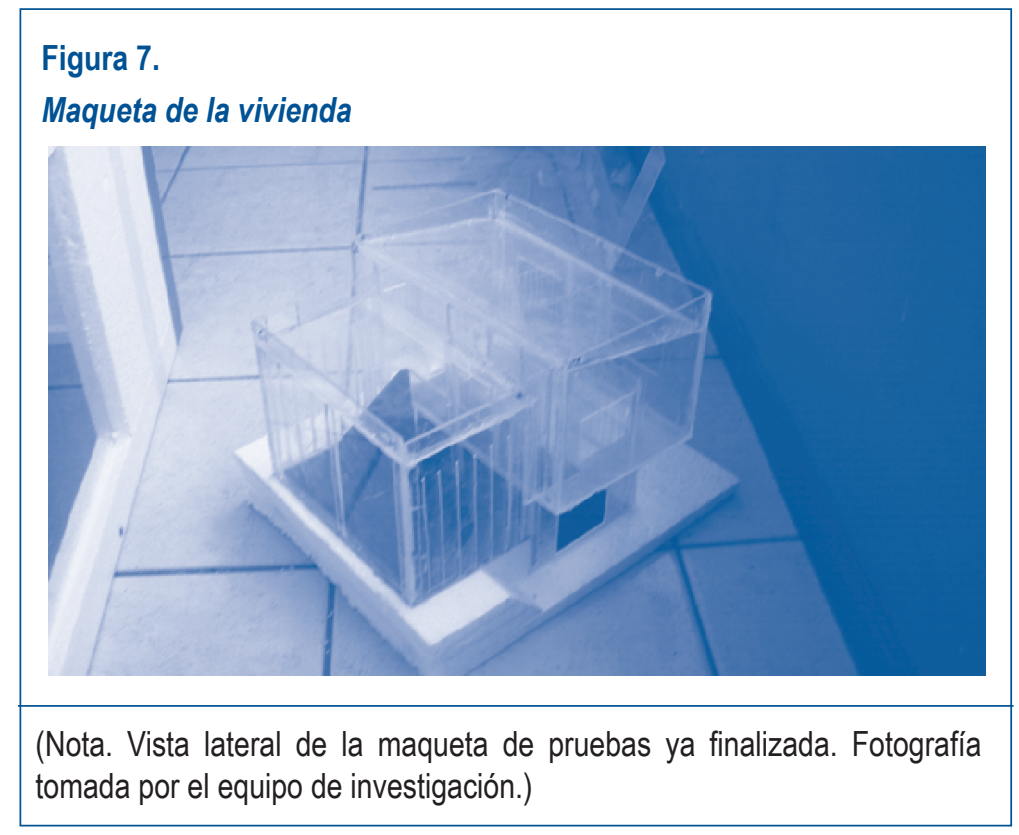

\section{Túnel de viento}

El túnel de viento con el que se realizó el experimento fue construido con poliestireno expandido de $3 / 4$ " de grosor y tres ventanas o escotillas de acrílico de $1 /{ }_{8}^{\prime \prime}$ de grosor -una en la parte superior y una en cada uno de los laterales-. Las ventanas o escotillas se construyeron colocando piezas de acrílico entre dos piezas de poliestireno expandido, sujetas entre sí con pegamento y silicón, que posteriormente fueron atornilladas entre sí para conformar el túnel. Para sellar el túnel de las posibles fugas de aire, se colocó cinta adhesiva en la parte exterior y silicón en el interior.

En el extremo que serviría como entrada del aire, se colocó una rejilla para conducir el aire de una manera más uniforme. Esta rejilla está también hecha de poliestireno expandido 
de $3 / 4$ " de grosor y consiste en una serie de huecos hechos con base en una cuadrícula de 1 por $1 \mathrm{~cm}$.

El aire se representó por medio de humo, producido por una máquina comercial de humo de 1.000 watts, que produce $5.000 \mathrm{ft}^{3} / \mathrm{min}$ de humo. La velocidad del flujo de aire fue reproducida por un ventilador semi industrial de aspas metálicas y tres velocidades, 18" de diámetro, 220 watts y una frecuencia de $60 \mathrm{~Hz}$. Se utilizó el ventilador, pues luego de varias pruebas se observó que el humo de la máquina tendía a concentrarse dentro del túnel, lo que reducía visibilidad; por lo tanto, la máquina de humo se colocó detrás de la rejilla de poliestireno expandido y detrás de la máquina el ventilador para empujar el humo producido por esta.

Para recolectar los datos se utilizó una cámara fotográfica con una resolución de 12 mega-pixeles, con rondas de disparo de tres cuadros por obturación a luz natural, con la que se obtuvieron fotografías sin ningún tipo de efecto para el proceso de construcción de la maqueta y el túnel de viento. Para el caso de los experimentos en sí, con la misma cámara se obtuvieron videos cortometrajes -que son muestras multimedia del experimento- en los que se realizó un proceso de selección de cuadros.

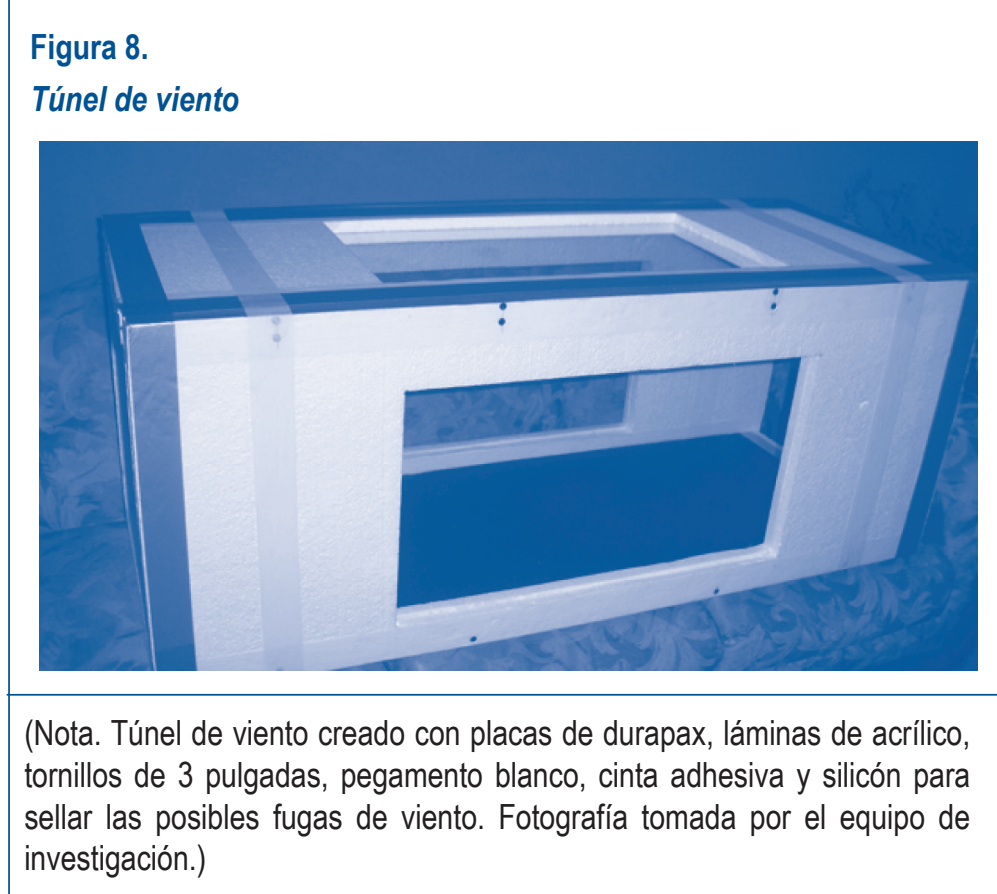

\section{Resultados y discusión}

Debido a una posible falta de claridad en los cuadros tomados de los videos, se consideró necesario presentar además de manera esquemática los resultados de las pruebas realizadas en el túnel de viento. Esto permite una mayor claridad y comprensión del comportamiento del flujo del aire dentro de la vivienda propuesta. 
Figura 9.

Ejemplo de uno de los esquemas del flujo del aire dentro y fuera de la vivienda

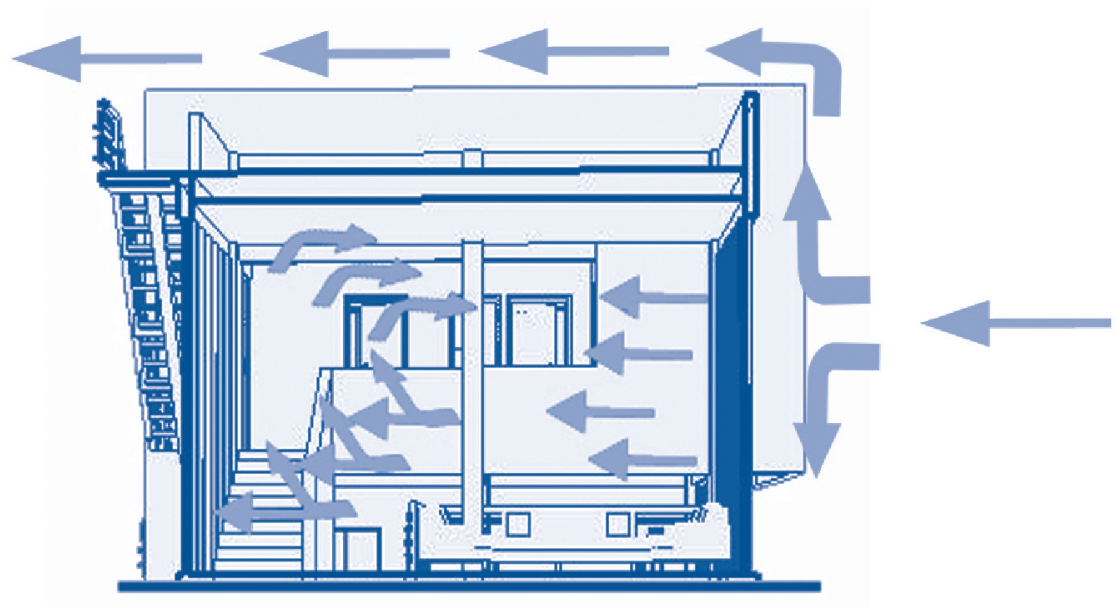

(Nota. La figura muestra cómo es la circulación del aire dentro de la vivienda y fuera de ella, vista de manera frontal, en las áreas de uso común. La celosía y la planta abierta permiten un flujo constante de aire hacia el interior, que circula libremente en todas las áreas expuestas. Elaboración propia.)

\section{Conclusiones y recomendaciones}

1. En principio, todo diseño debe considerar los condicionantes del lugar donde se emplazará la edificación. Sin embargo, por distintos motivos económicos, sociales, culturales, entre otros- estos criterios han sido dejados de lado en la actualidad. El diseño de una vivienda bioclimática y sostenible es logrado a través del proceso de investigación de los condicionantes del lugar de emplazamiento y la identificación de las opciones sobre tecnología aplicada, pero a la vez plantea y se relaciona fuertemente con un compromiso de solidaridad para con los usuarios y el medio ambiente natural en el que estará ubicada.
2. La vivienda propuesta trata de integrar soluciones constructivasqueaprovechen los recursos naturales de los que se dispone en el país, tales como el viento, el asoleamiento y las precipitaciones pluviales. Así, se hace uso de elementos como colores, vanos y ventanas, estructuras específicamente diseñadas para el aprovechamiento de los recursos antes mencionados, etc.

Sin embargo, para garantizar la viabilidad de la propuesta es imperante continuar realizando pruebas, algunas de mayor rigurosidad. Entre las pruebas que se considera necesario continuar realizando se encuentran: 
- Pruebas relacionadas con las precipitaciones pluviales y el almacenamiento del agua por medio de cisternas.

- Pruebas en cuanto a la viabilidad real y otros aspectos relacionados con la instalación del sistema de paneles fotovoltaicos para proveer de energía a la vivienda.

- Pruebas relacionadas con aspectos estructurales y de materiales.

- Pruebas relacionadas con la temperatura interna de la vivienda propuesta.

3. La bioclimatización y sostenibilidad de una vivienda es posible, pero depende de la inclusión de ciertos recursos que no son habituales en el ámbito constructivo del país. En la actualidad no es posible que todos los estratos socioeconómicos alcancen los beneficios tecnológicos, pues se requieren ciertos recursos económicos para hacer posible su implantación en los diseños. Sin embargo, con esto, no se descarta la posibilidad de hacer llegar los beneficios de la arquitectura bioclimática y sostenible a los estratos sociales más bajos.

\section{Referencias}

- Allen, E.; Iano, J. (2002). El anteproyecto arquitectónico. Guía para su ejecución. México: Editorial Limusa, S.A. de C.V.

- Ayala, M.; Sánchez, I.; Escalante, A.; Marroquín, W. (2005). "Determinación del potencial solar y eólico en El Salvador". San Salvador: Ministerio de Medio Ambiente y Recursos Naturales (MARN), Universidad Centroamericana "José Simeón Cañas" (UCA), Servicio Nacional de Estudios Territoriales (Snet). Recuperado de http://mapas.uca.edu. sv/swera/

- García, L. (2009). "Régimen de Iluvia y caudales en El Salvador, su relación con la variablidad climática (forzantes oceánicos-atmosféricos) para la construcción de mapas de amenazas por déficit o exceso de lluvias" (Tesis de Maestría, Ciudad Universitaria Rodrigo Facio, Costa Rica). Recuperado de http://portafolio.snet.gob.sv/digitalizacion/pdf/spa/ doc00254/doc00254.htm

- Higueras, E. (2006). Urbanismo bioclimático. Barcelona: Editorial Gustavo Gili.

- Muñoz Veneros, D. (2003). "Arquitectura ecológica sustentable". Recuperado de http://www.eco2site.com/arquit/entrevista-dante.asp

- Olgyay, V. (2002). Arquitectura y clima. Manual de diseño bioclimático para arquitectos y urbanistas. (2a . Ed.). Barcelona: Editorial Gustavo Gili.

- Serra, R. (1999). Arquitectura y climas. Barcelona: Editorial Gustavo Gili.

- Servicio Nacional de Estudios Territoriales, Servicio Hidrológico Nacional (2005). "Balance hídrico integrado y dinámico en El Salvador. Componente evaluación de recursos hídricos". Recuperado de http://www.snet.gob. sv/Documentos/balanceHidrico.pdf 